\title{
EDUCAR: VALIDEZ Y VIABILIDAD DE LA CONSTITUCIÓN DE UN NUEVO SIGNIFICANTE
}

\section{EDUCATE: VALIDITY AND VIABILITY OF THE CONSTITUTION OF A NEW SIGNIFIER}

\section{ANTONIO SANTOS RUMBEA', MÓNICA FRANCO POMBO²}

1 Universidad Católica de Santiago de Guayaquil. antonio.santos@cu.ucsg.edu.ec

$\underline{2}$ Universidad Católica de Santiago de Guayaquil. monica.franco@cu.ucsg.edu.ec

palabras clave: Educación, Psicoanálisis, Discurso, Significante, Amo, Universidad, Modernidad, Posmodernidad, llustración, Teoría, Crítica, Freud, Lacan, Foucault, Habermas.
RESUMEN

En este texto se presenta una parte de los resultados de la investigación sobre el Análisis del discurso de los actores del sector educativo con respecto a la educación como praxis, que tomó como objeto de estudio las prácticas discursivas de los actores de dicho sector. Este artículo se centra en la validez y viabilidad de constituir un nuevo significante para el signo educar. ¿Es posible pensar en otra lógica para el discurso de la educación; lógica cuya referencialidad sea una ruptura con cierta parte de su historia? Desde una reflexión que asume principalmente la perspectiva psicoanalítica lacaniana y que incorpora referencias del pensamiento filosófico, se logra estructurar un análisis de las líneas discursivas que llevan a una respuesta afirmativa al interrogante de inicio pero que, fundamentalmente, da cuenta de la necesidad de resignificar los signos lingüísticos asociados comúnmente con la práctica educativa.

La hipótesis fue que la formulación de un nuevo significante en la experiencia psicoanalítica postula efectos en los sujetos en cuanto que constituye una relación y una praxis inédita del saber que para el caso tendría consecuencias de reinvención del propceso educativo.
This text presents part of the results of the research on the analysis of the discourse used by actors of the educational field, with respect to education as the praxis that took as a subject of study the discursive practices of said actors. This paper focuses on the validity and feasibility of the process of constituting a new signifier for the sign "Educar". Is it possible to think of another logic for the discourse used in the field of education; logic whose referentiality is a divorce from a certain part of its history? The approach of this topic throughout this paper is based on a reflection that takes the Lacanian psychoanalytic perspective and that includes references of philosophical thought. By taking this approach, it is possible to structure an analysis of the discursive lines that lead to an affirmative answer to the initial question but, fundamentally, features the need to resignifying the linguistic signs associated with educational practice. The stated hypothesis is that the formulation of a new signifier in the psychoanalytic experience causes effects in the subjects insofar as it constitutes a relation and an new practice of knowledge that has consequences of reinvention of the educational process.

KEYWORDS: Education, Psychoanalysis, Discourse, Idea, Master, University, Modernity, Postmodernity, illustration, theory, Critics, Freud, Lacan, Foucault, Habermas. 


\section{INTRODUCCIÓN}

Sigmund Freud, quien creó el Psicoanálisis, planteaba que hay tres profesiones imposibles: educar, gobernar y psicoanalizar (Freud, 1932: 3361). Ese no-posible podría ubicarse desde la aparente insuficiencia de los resultados en el caso de estas profesiones, dado que se enmarcan en una línea aspiracional que compite, casi siempre, con un ideal: el ideal del hombre que debe formarse, el ideal de hombre que vive y ese ideal de sujeto hombre que debe gobernar para el bien común.

Por medio de entrevistas en profundidad, grupos de enfoque y encuestas, se abordaron las variables a investigar de modo que se posibilitara el registro de las variantes discursivas; proceso que permitió un análisis y formulación de lineamientos de reflexión de la hipótesis en docentes y estudiantes de universidades y unidades educativas particulares y estatales en la ciudad de Guayaquil.

De otro lado, el estudio implicó aspectos teóricos orientados a fundamentar el acto educativo formal, lo que se concretó en:

- La identificación de una doxa en cuanto a los discursos efectivos vinculados con la realización de las performances educativas.

- El análisis de la doxa para identificar determinaciones ideológicas como sistemas de valores y creencias.

- El análisis de las idealizaciones y de las posibles aporías y callejones sin salida con sus efectos y consecuencias.

Con base en los objetivos anteriores se pudo realizar una aproximación conceptual que tomó estos resultados como elementos para fundamentar una teoría de la educación posible.

\section{TIPOLOGÍA DE DISCURSOS VINCULADOS A LA EDUCACIÓN}

Para el estudio investigativo se seleccionaron varios aspectos conceptuales que orientaron el proceso de análisis de los discursos de los actores del proceso educativo y la validez y viabilidad de la constitución de un nuevo significante en torno a educar.

LOS DISCURSOS Y SU ESTRUCTURA

Tanto para el psicoanálisis como para otras perspectivas como las que plantea Michel Foucault, el discurso no solamente es un hecho del habla; sin duda del hablante, del ser sujeto del lenguaje, sino todavía más, da cuenta del orden simbólico que es propio de lo humano.
En el marco analítico de Foucault, el discurso constituye un concepto central. Los discursos se refieren a lo que puede ser dicho y pensado, pero también a quién puede hablar, cuándo y con qué autoridad. Los discursos producen significación y ciertas relaciones sociales; construyen tanto la subjetividad como las relaciones de poder. Los discursos son "prácticas que configuran sistemáticamente los objetos de los que hablan... Los discursos no se refieren a objetos; no identifican objetos; los construyen y, al hacerlo, ocultan su propia invención" (Foucault, 1974, p.49).

Por tanto, las posibilidades de significado y de definición están cubiertas de antemano por la posición social e institucional de quienes se hacen como sujetos en ellos. Así, los significados no surgen del idioma, sino de las prácticas institucionales, de las relaciones de poder. Las palabras y los conceptos cambian su significado y efectos según el discurso en el que se despliegan.

Los discursos limitan las posibilidades del pensamiento. Ordenan y combinan las palabras en formas específicas y excluyen o desplazan otras combinaciones. Sin embargo, en la medida en que los discursos se construyen por exclusiones e inclusiones, por lo que no debe decirse tanto como por lo que sí puede ser dicho, mantienen relaciones antagónicas con otros discursos, otras posibilidades de significado, otras peticiones, derechos y posturas. Este es el principio de discontinuidad que formula: "Hemos de tener en cuenta los poderes complejos e inestables por los que el discurso es a la vez instrumento y efecto del poder pero también obstáculo, escollo, punto de resistencia y punto de partida para la estrategia opuesta" (Foucault, 2010, p.101).

En principio se afirma que el discurso implica lazo social, es decir, una comunidad con la que existe referencialidad y al mismo tiempo regulación. Es una estructura establecida con base en un orden simbólico, entendiendo que el lenguaje permite la formalización y la postulación de una realidad.

El "modelo comunicacional" del conocimiento destaca el argumento de que no tenemos un acceso irrestricto a las entidades del mundo, independientemente de nuestras prácticas para alcanzar el entendimiento y del contexto lingüísticamente constituido de nuestro mundo de la vida: Los elementos de lo que llamamos 'lenguaje' o 'mente' penetran tan profundamente en lo que denominamos 'realidad', que el mero proyecto de representarnos a nosotros mismos como 'manejadores' de algo 
'independiente del lenguaje' está fatalmente comprometido desde el principio (Habermas, 2007, p.9).

En su decimoséptimo seminario que llamaría El reverso del psicoanálisis, Lacan plantea la concepción de los cuatro discursos. Asume la formulación del discurso y establece una estructura en la que configura un matema, como recurso explicativo, constituido por cuatro términos, en el sentido de las agujas del reloj: el agente, el Otro, la producción y la verdad. La vertiente de matematizar es el intento de evitar el malentendido.

$\frac{\text { AGENTE }}{\text { VERDAD }} \longrightarrow \frac{\text { OTRO }}{\| \text { PRODUCCIÓN }}$

Así entre el agente y el Otro la flecha denota la imposibilidad: el agente no logra llegar hacia el Otro, primero porque siempre es un semblante y su verdad no puede ser cabalmente formulada para el Otro y porque el Otro con mayúsculas, que implica al orden simbólico, lo registra en sus términos.

El postulado de la comunicación se encuentra con la imposibilidad: si pretendo comunicar una historia, comunicar mi experiencia, el Otro la registra en sus propios términos. El desfase no es por un mal-entendido, sino por un entendimiento que siempre es no-todo además por asumir neutralidad en el Otro. El lugar del agente implica una dimensión de semblante en tanto la posición desde donde asume el discurso es solo una dimensión del mismo. El psicoanálisis formula que el efecto no es de comunicación sino de desplazamiento del discurso. El discurso se desplaza cuando de una posición en una estructura discursiva, el sujeto asume un giro a partir de la experiencia y su componente de lazo social.

Entre la verdad y la producción, el doble signo barrado, implica el concepto de impotencia: la producción, lo que postula efectos en el discurso, no logra esclarecer lo que hace función de verdad. Verdad que es un lugar lógico que realmente lo determina como causa.

Al mismo tiempo define cuatro conceptos que expresa por una letra, para clivar entre las posiciones: el Significante-Amo (S1), el Saber (S2), el Sujeto (\$), el objeto a o plus de goce (a). Sus ubicaciones delimitarán cuatro discursos como hechos de estructura, los discursos del Amo, de la Universidad, de la Histérica y del Analista.

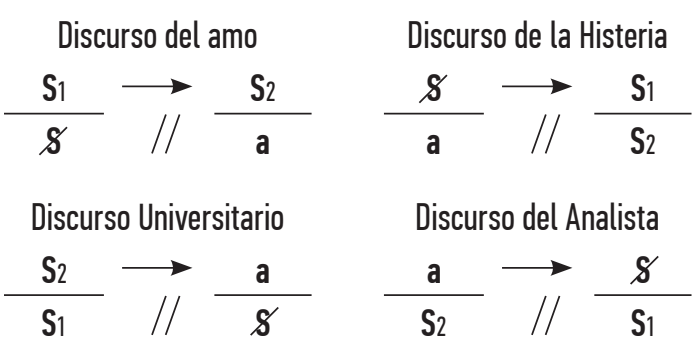

En el matema del discurso de la Universidad, el S2 es el saber. Formula que en una cadena significante existe una secuencia que se inicia con un significante que es punto de partida formal (S1), al que Lacan llama significante Amo. Y en el proceso, desde ese punto de partida, se constituirá una derivación que constituye al saber. Así en el lugar del agente se encuentra el saber, la afirmación de lo que se cree saber o de loque efectivamente se sabe. Antes ese saber en occidente era atesorado en los monasterios; a partir del siglo XII las universidades inician un proceso de establecimiento y regularización del mismo. Proceso que originalmente estará alineado hacia un nuevo ejercicio del poder.

En la posición del Otro está aquello que es destinatario del discurso. En el psicoanálisis el objeto "a" o plus de goce es el nombre de la huella que el proceso de represión condiciona en los sujetos. Freud llamaba castración a la sustracción que permite establecer, por una parte, el no-todo, la condición deseante, que se denota incompletud y que al mismo tiempo establece lo que Lacan llama un plus de goce.

El concepto de goce en psicoanálisis no se vincula con el placer, más bien todo lo contrario. En ese orden no debe ser entendido en la acepción común, sino más bien en la línea de la patior, vinculada con el origen del término pasión: aquello que abisma, lo que hace padecer.

De ese modo, la sujeción permite que el objeto causa del deseo que es el "a", filtre el goce, vinculado con la pulsión de muerte, obteniendo tan solo su plus; no el goce en cuanto tal, sino solo un residuo. Un residuo que está hecho en el conjunto de la estructura. Ya lo sabemos con el modelo del cubo de Rubik: los intervinientes son mutuamente solidarios. La letra "a" simplifica e intenta, como tan solo letra, simbolizar al otro (autre en francés). Esta "a" tiene unas consistencias mutuamente solidarias. Esta "a" tiene una consistencia se encuentra, con la dimensión objetiva del estar ahí, pero a su vez, ser del orden de lo imposible, de simbolizar en sí solo de un modo aproximado y evanescente. Aquel objeto, que Lacan postula como el del psicoanálisis. 
En el lugar de la verdad está posicionado el S1, el llamado significante amo, por alusión a la dialéctica hegeliana del amo y del esclavo. En Saussure el sentido de un significante depende de los otros significantes. Si hay S1 es porque hay $\mathrm{S} 2$.

Un significante no es sino en cadena. Una historia no es, sino se estructura vinculantemente con otras historias. A este significante de punto de partida también se lo postula como significante Amo. El que se encuentre en el lugar de la verdad expone una voluntad de poder y de referencialidad; como todo poder, no podrá evitar lo mortífero y lo siniestro en sus consecuencias. Si bien es cierto el discurso de la Universidad no es el discurso del Amo, sin embargo le sirve. El S1 quedará representado en el lugar del agente por el S2, el saber. Siendo que el S1 es lo que lo origina, en general, una pregunta, que quedará elidida, representada por el saber (S2). La pregunta no es lo que se evalúa en el Discurso Universitario, su pertinencia, consistencia, relevancia, fundamentación; se evalúa el S2, la respuesta.

El discurso de la Universidad produce un sujeto tachado (\$), esa producción implica efectos.

La idea de Lacan en cuanto a la letra $S$ barrada (\$) es múltiple, por una parte se vincula con las S1 y S2 en cuanto dimensión significante. Dirá que un significante es lo que representa a un sujeto para otro significante. Podemos decir, el sujeto es el efecto de una historia o mejor de un proceso de historización. En este caso, el discurso produce un sujeto, un sujeto tachado, en el irrefutable sentido de sus carencias, de su falla que en psicoanálisis se denomina castración. La cifra que se escapa del resultado y que no permite la totalización del orden de un saber absoluto, de un saber total, de un saber con certeza, de un saber enloquecido. En las psicosis se encuentra ese saber en los cuadros delirantes, fundamentalmente paranoicos.

Finalmente digamos que el discurso de la Universidad es el contrapunto del de la ciencia. Ciertamente no hay que imaginar que el docente que enseña matemáticas es el que establece teoremas o ecuaciones en matemáticas puras o aplicadas. Igualmente para con las otras ciencias que toma para su divulgación.

Recordaremos la idea de la tecnoburocracia para decir que las tecnologías son articulaciones de saber con fines operativos y utilitarios, su respuesta es: esto sirve para..., lo otro, lo que claramente no sirve, que espere. Así el discurso del Amo, es absolutamente indiferente con la subjetividad al punto que, su variante, el discurso del capitalismo, omnicomprensivo y todopoderoso a la fecha, con sus unidades de intervención, entre ellas las ciencias, postulan que los sujetos, de verdad, no interesan y es más, son una cuestión a objetivar.

El discurso del Amo les plantea a los sujetos hacia dónde deben ir. Para ir hacia allá han de sacrificarse, sacrificar lo que realmente son. Y en cuanto a sus deseos, que esperen sentados. No en balde el psicoanálisis aparece en estos tiempos, en los que el sujeto está tan perdido en relación con lo que causa su condición, como sujeto deseante, no-todo. No es igual a lo que le ponen en frente, como la zanahoria al buey, para hacerlo arar.

Sostenemos que el proceso de producción de los discursos didácticos - "regímenes de verdad" en el sentido foucaultiano de la expresiónes inseparable e incomprensible sin prestar atención a las condiciones económicas, sociales, culturales y políticas que contribuyeron a esculpir las funciones de la escuela como institución de control dedicada tanto a la producción de sujetos como a la reproducción social y cultural en la era del capitalismo. Recordar qué son los sistemas escolares, de dónde provienen y cuándo inician sus despliegue, en franca coincidencia temporal con el de los estados nacionales del capitalismo, es como anunciábamos, requisito previo y obligado para reconocer y comprender el lecho magmático en donde se fueron gestando los primeros cuerpos de doctrina metodológica acerca de muy distintas materias de conocimiento destinadas, fundamentalmente, al adiestramiento y sometimiento de la materia y espíritu de una infancia continuamente recreada. (Mainer, 2009, p.23).

El discurso de la educación se ufana de poder hablar en nombre de la ciencia, o al menos de algunas de sus consideraciones; siendo por una parte que divulgar no implica otra cosa que un ejercicio de simplificación y por otra, desesperado por la bendición del Amo, a fin de cuentas los presupuestos que su lógica permite. Dicho discurso del Amo afina la especialidad y la especialización por múltiples determinaciones, quizás las más importantes son la de poder: la ausencia de visión global permite compartimentar los espacios de saber para articularlos con fines de uso. 
El Amo oculta su falta, su falla, su endeblez y la viste de mil maneras de modo que evita evidenciar su fragilidad.

Por eso aquí formulamos que el discurso de la educación en la antigüedad, estaba más dentro del orden del Amo, que de ser una variante del de la Universidad

Así el Discurso del Amo procura ordenar un mundo en su coherencia programática. Ciertamente el saber implica poder, pero no solo, el saber implica poder porque es un saber que se fundamenta en la demanda de efectos.

Sin embargo, lo que se produce es el objeto a, evanescente, inasible. Un amo está sujeto a su esclavo, tanto como su esclavo puede estar de otra manera determinado por él. ¿No es, por ejemplo, en esta época de la tecnología, aquello de lo que creemos ser amos, realmente nuestro dueño? Solo basta unos minutos de corte eléctrico para saber bien quién es quién.

En esta estructura, el lugar de la verdad, si Lacan lo sitúa bajo la barra del agente, es también para expresar que siempre será aquello de lo que no se quiere saber nada. En este caso de su tachadura (\$), de que el Amo, tras el traje, está también desnudo; como lo planteaba Hans Christian Andersen en su cuento El traje nuevo del emperador, conocido como El rey desnudo.

\section{VALIDEZ Y VIABILIDAD DE LA CONSTITUCIÓN DE UN NUEVO SIGNIFICANTE}

\section{Dar lo que no sabemos a alquien que no somos.}

El dicho parafrasea la expresión que puede ser planteada en cuanto al efecto de un nuevo significante de educar, en el que la posición del sujeto asume a la ignorancia como pasión. El maestro zen no responde, al menos no directamente o si lo hace adviene lo imprevisible. Así la constitución de efectos en el discípulo, en cuanto a concernirse con la formulación de las respuestas posibles. El Otro no es garante sino que es espacio de reconocimiento del proceso de significación. No hay saber solitario, como no hay autoanálisis. Un discurso siempre implica un lazo social. Ese lazo social no tiene que ser de contenidos establecidos, es de comunicación en el orden de la interacción, por así llamarla, que puede llevar auna rectificación o a una reformulación.

Dar lo que no sabemos: ¿cómo es posible? La condición deseante está establecida a partir de una falta. El discurso del Amo procura, digámoslo así, superarla. En psicoanálisis el concepto de fantasma establece una experiencia en que dicha falta se encuentra suturada, se encuentra engañosamente resuelta. El fantasma tiene visos de realidad pero lo que lo sostiene no es más que una fantasía inconsciente, es decir, una fantasía que no se reconoce como tal. Indiquemos que el discurso procura una derivación que nominamos proceso de significación. En ese proceso se establecen los sentidos. Pero no hablamos que la articulación de un significante con otro, que realmente es con otros, puesto que son en conjunto, no produce un sentido, sino sentidos. El Yoprocura estabilizar el proceso delimitando el ámbito de la significación y estableciendo un sentido dominante.

Si el proceso se engancha con un sentido dominante o hasta con el sentido Uno, deviene la estabilización fantasmática que procura un orden unívoco.

Así el saber, como el efecto de la estabilización de la deriva significante, obstruye precisamente la dinámica y eso por una opción de poder que tiene como objetivo conducir y lograr efectos. En cuanto a las subjetividades en tanto tales, no las tiene en cuenta, salvo en la variante de servirse de ellas en lo formal para lograr los objetivos de control.

Por otra parte, el saber que no se sabe es el del inconsciente, ese otro discurso. Hay algo de lo que los sujetos no quieren saber y todavía más evidentemente en una época que hace del culto de los bienes, del utilitarismo, del pragmatismo su leitmotiv.

Este nuevo significante implicará efectos de construcción de un sujeto, como la fórmula dar lo que no se tiene a alguien que no se es, determina el efecto de la constitución de un nuevo significante del amor, al haber pasado el sujeto por el dispositivo psicoanalítico, lo que implicará efectos de transformación subjetiva y de posición discursiva.

¿Qué es lo que podría en el ámbito personal llevar hacia esta transformación? En principio el pausado esclarecimiento de lo que nos ha conducido hacia nuestra elección como educadores y, por qué no, el compromiso por un análisis personal. Existe una historia de la que somos sujetos y en la medida en que esa historia no esté formalizada, nuestras elecciones son solo síntomas privilegiados, es decir, malentendidos.

Lo que es causa del saber no es el saber mismo, en ocasiones más bien es la ruptura con un saber establecido. Lo que es causa del saber, es 
ausencia, falla, discordancia que permite el acto de preguntarse, de cuestionarse. No porque la concordancia sea posible, sino porque son posibles diferentes formas de ella.

Así la pulsión de muerte procura la supresión del saber por medio de un saber absoluto. Por saber entendemos al proceso de elaboración permanente que da cuenta de formas distintas de formular la realidad. Hemos llamado distintas, por no decir nuevas, ya que la noción de novedad es utilizada y propiciada por el discurso del Amo en su vertiente capitalista, para barnizar en la experiencia social y subjetiva, efectos de proliferación que implican realmente al ciclo de la circulación de la mercancía y la necesidad de que la curva de consumo, de ingresos sobre los egresos, de la acumulación, sea siempre ascendente.

Formas de formular la realidad que no están supeditadas a la dimensión de lo absoluto que implica la respuesta correcta.

MATERIALES Y MÉTODOS

El estudio se formuló desde un fundamento teórico, que enmarcó el registro y el análisis de los discursos efectivos en cuanto realización formalizada por medio de la palabra. Este estudio se sostuvo con base en las referencias conceptuales que provienen básicamente de tres ámbitos:

- La teoría psicoanalítica lacaniana. El psicoanalista Jacques Lacan asume una reformulación del discurso psicoanalítico al que evidenciaba en un proceso de desfundamentación con base en demandas alienadas a la ideología del bienestar y determinadas por concepciones ideológicas de salud y enfermedad. Así el proyecto lacaniano es retomar las fuentes freudianas en las que evidencia un conflicto con formulaciones adaptativas y manipuladoras. Formalizaciones que implicarán impactos no solo en el ámbito del psicoanálisis sino también en la cultura y ámbitos del saber.

- El análisis del poder. Son las formulaciones que abordan el análisis de las determinaciones e implicaciones del poder. Los planteamientos de Michel Foucault se abren con múltiples implicaciones que aportan hacia la fundamentación de una teoría de la educación que reflexione y alcance efectos sobre el saber como ejercicio del poder. Todos los sistemas educativos se fundamentan en un saber del cual hacen punto de partida. La reflexión sobre otras dimensiones desde donde resignificar la educación deben llevar a una transformación no solamente formal o de procedimientos, sino a una transformación sustantiva. En la tradición filosófica occidental podemos encontrar referentes de ello por ejemplo en el pensamiento a raíz de la Ilustración.

- El anÁlisis FIlosófico QUE dimensiona MODERNIDAD Y POSTMODERNIDAD. Este análisis evidencia a unas praxis que enfatizan lo instrumental por sobre lo sustantivo que queda relativizado y hasta ironizado por los discursos que exponen como emblemas los indicadores y los márgenes, a fin de cuentas francamente el retorno y la rentabilidad, la utilidad.

La investigación empírica se planteó desde una metodología mixta CUAL-CUAN, en la que la dimensión cualitativa se asumió para desencadenar, detectar y registrar los discursos objeto de estudio; siendo que estos discursos, al afirmarse, también constituyen de suyo el ámbito de lo que ocultan.

En ese sentido, una intervención y análisis del discurso desde una articulación psicoanalítica nos permitió apreciar otros registros e implicaciones.

Por medio de entrevistas en profundidad y grupos de enfoque se trabajaron las variables a investigar de modo que se posibilite el registro de las variantes discursivas, proceso que permitió un análisis y formulación de lineamientos de despliegue de la hipótesis. La fase cuantitativa que procuró mensurar los hallazgos que la fase cualitativa y su análisis proveyó.

El estudio cualitativo concentró acciones en directivos, docentes y estudiantes de educación Básica Superior y Bachillerato ubicados en la ciudad de Guayaquil en la República del Ecuador.

Las entrevistas en profundidad fueron realizadas con las autoridades y los docentes.

La riqueza de los grupos de enfoque permitió recrear las interacciones entre los estudiantes.

Fue posible evidenciar tendencias y otros aspectos vinculados con las dimensiones en juego, que permitieron, por medio de un análisis de la información, elaborar conclusiones.

ANÁLISIS DE LOS RESULTADOS

Que el saber sea estructurado a partir del no saber implica las siguientes consideraciones para la teoría de la educación: 
- El educador no solo valora la posición del educando aún más, le es fundamental, no como un acto de empatía o de franca generosidad, sino como una apertura hacia la elaboración de un saber en construcción. El niño que se enfrenta a las vocales y, en general a la lectoescritura, qué percibe frente a ellas: son unas marcas, la letra $a$ será un globo con cola. No se trata de lo anecdótico, se trata de empoderar las percepciones, las palabras, elaborarlas y desplegar el saber que está ahí. Hasta llegar probablemente a qué es la letra a como letra y suscitar la investigación al respecto, entendiendo siempre lo lúdico como una dimensión del aprendizaje. Una vez que llega a saber lo que son las letras es porque parecería haber adquirido la conciencia fonológica.

- El educador es una posición formal, importante en cuanto a su función suscitadora y no intimidante. Una posición que asume que en el proceso con el educando, ambos son educados, que existe un aprendizaje en común, que puede ser diverso. Lo en común no implica la homogeneidad sino la experiencia de aprender. Al mismo tiempo, que el aprendizaje en común no es solo de contenidos, sino de la relación, del otro, de sus particularidades no estandarizables. El aprender nos transforma, hace lo que todo alimento, el cual no se limita tampoco a "mantener»: como sabe el fisiólogo. Pero en el fondo de nosotros, totalmente "allá abajo», hay en verdad algo rebelde a todo aleccionamiento, una roca granítica de fatum (hado) espiritual, de decisión frente a preguntas predeterminadas y respuestas elegidas (Nietzsche, 2015, p.352).

- La posición del educador se fundamenta en una regulación, que a similitud de la regla freudiana de la asociación libre, es el punto de partida y el compromiso de los intervinientes. Regla que se puede formular así: las respuestas son correctas no en sí mismas sino en la medida en que las preguntas son ensayadas de distintas maneras. Nuestro compromiso está en dilucidar las preguntas y las diferentes formas de entender un problema o situación. Frente a un saber, nuestro deber es delimitar lo que no se sabe. Si vamos a estudiar al Sol como estrella, preguntémonos qué no sabemos del Sol. Sin duda implica una investigación con respecto a lo que sabemos, pero la orientación, la perspectiva, el carácter del proceso supone una impronta de falla y no de poblar con afirmaciones un espacio discursivo mientras barremos bajo la alfombra lo que no calza.

- El proceso conduce a relativizar la verdad, o el resultado, dando importancia al proceso en sí mismo. Debemos entender que la cultura del pragmatismo y del utilitarismo empodera los resultados y al proceso en la medida en que los logra; de ahí la frase el fin justifica los medios. Debemos entender que es una simulación a la cual podríamos responder con el refrán de que no por mucho madrugar amanece más temprano. Cierta posición pragmática igualmente cuestiona a la verdad, hasta logra excluirla al ámbito de lo verosímil. No es una historia nueva, se originó con los Sofistas. Su sentido es lograr que los resultados no se vinculen con ningún aspecto que pueda parecer del orden de la ética. Es un aspecto crucial al que el discurso de las ciencias se ha plegado puesto que siempre, hoy como ayer sobre todo en ciertas ciencias, necesitó de los mecenas, menos tiempo que el arte es cierto, como hoy del capital.

Relativizar la verdad no es porque se la excluya o se la convierta en verosimilitud, sino porque ha de postularse una nueva manera de realizarla que la formulamos: la verdad es el momento del caos de una certeza o afirmación dominante.

El problema de la educabilidad ha sido tratado, en la vieja literatura, con objeto de saber si la educación es posible o bien si ha de estrellarse contra la naturaleza, innata e invariable, del carácter y la constitución. El pesimismo pedagógico ha declarado con frecuencia que toda labor educativa es un "trabajo de Sísifo" y un piadoso engaño de sí mismo, nula, a fin de cuentas, en sus efectos, ya que, o bien apoya la naturaleza, ya de sí un tanto impulsiva, y entonces es superflua, pues el hombre será sólo aquello que ya es y tal cual es, o bien la cubrirá de un barniz de cultura, la ocultará bajo un disfraz, y en este caso no existe garantía alguna de que todo este trabajo no se vea destruido por un desbordamiento de la naturaleza verdadera e 
indestructible. "Descender de nobles", ser de buena raza y condición, sería más importante que ser educado. (Mialaret, 1984, p.75).

CONSIDERACIONES FINALES

Estás formulaciones establecen los rudimentos de lo que podría ser una nueva teoría de la educación. Teoría que se fundamenta en el no ser, en la construcción permanente de la pregunta; en el entendimiento de respuestas provisionales. No aspira modelar al Otro con base en un ideal. Puede ser postulada con la frase: dar lo que no sabemos a alguien que no somos. Implica el análisis profundo de nuestra posición de educadores en cuanto a las determinaciones subjetivas que nos conducen ahí, de manera de que no sea simplemente un síntoma no analizado.

Así como en la física existen los ámbitos teóricos y experimentales, y están bien delimitados; esperamos que advengan aquellos que lleven a cabo la realización de estos planteamientos que se fundamentan en la posibilidad de la formulación de un nuevo significante, dígase, una nueva forma de advenir del sujeto en la educación.

\section{REFERENCIAS BIBLIOGRÁFICAS}

Foucault, Michel (1974) Michel Foucault, El orden del discurso, Tusquets, Barcelona.

Foucault, Michel. (2010). Obras esenciales. Segunda parte: Estrategias de Poder. Madrid. Paidós.

Freud, Sigmund (1932) Nuevas lecciones introductorias al Psicoanálisis. Obras completas tomo III. Madrid. Editorial Biblioteca Nueva.

Habermas, Jürgen. (2007) Sobre la verdad ¿validez universal o justificación?. Buenos Aires. Amorrortu.

Lacan, Jacques (2008) Seminario 17: El reverso del Psicoanálisis, Buenos Aires, Ediciones Paidós.

Mainer Baque, José Carlos. (2009) La forja de un campo profesional. Pedagogía y didáctica de las ciencias sociales en España (1900-1970). Madrid: Consejo Superior de Investigaciones Científicas.

Mialaret, Gastón. (1984). Diccionario de ciencias de la educación Barcelona. Oikos-Tau ediciones.

Nietzsche, Friedrich. (2015) Más allá del bien y del mal. Madrid. IberiaLiteratura 